\title{
Interpolation on Uniform Meshes By the Translates of One Function and Related Attenuation Factors
}

\author{
By F. Locher
}

\begin{abstract}
The exact Fourier coefficients $c_{j}\left(P_{n} f\right)$ are proportional to the discrete Fourier coefficients $d_{j}^{(n)}(f)$ if $P_{n}$ is a translation invariant operator which depends only on the values of $f$ on an equidistant mesh of width $2 \pi / n$. The proportionality factors which depend only on $P_{n}$ but not on $f$ are called attenuation factors and have been calculated for several operators $P_{n}$ of spline type. Here we analyze first the interpolation problem which is produced by the functions $\sigma(\cdot-2 \pi j / n), j=0, \ldots, n-1$, where $\sigma$ is a suitable $2 \pi$-periodic generating function. It is essential that the associated interpolation matrix is of discrete convolution type. Thus, we can derive conditions guaranteeing the unique solvability of the interpolation problem and representations of the interpolating function. Then the attenuation factors may be expressed in terms of the Fourier coefficients of $\sigma$. We point especially to the case where $\sigma$ is a reproducing kernel in a suitable Hilbert space. Here we get attenuation factors of a new type which are generated by interpolation with analytic functions.
\end{abstract}

1. Introduction. The Fourier coefficients

$$
c_{j}(f):=\frac{1}{2 \pi} \int_{0}^{2 \pi} f(t) \exp (-i j t) d t, \quad j=0, \pm 1, \pm 2, \ldots,
$$

of a $2 \pi$-periodic, real valued function $f$, whose values at the nodes

$$
t_{\mu}=\frac{2 \pi}{n} \mu, \quad \mu=0, \pm 1, \pm 2, \ldots,
$$

are known or can be calculated to a desired degree of exactness, are usually approximated by the discrete Fourier coefficients

$$
\begin{aligned}
d_{j}^{(n)}(f) & :=\frac{1}{n} \sum_{\mu=0}^{n-1} f_{\mu} \omega^{-\mu j}, \quad j=0, \pm 1, \pm 2, \ldots, \\
f_{\mu} & :=f\left(t_{\mu}\right), \quad \mu=0, \ldots, n-1, \\
\omega & :=\exp \left(\frac{2 \pi i}{n}\right) .
\end{aligned}
$$

These may be computed very efficiently by the algorithm of Cooley and Tukey (Fast Fourier Transform). As the values $d_{0}^{(n)}(f), \ldots, d_{n-1}^{(n)}(f)$ may be regarded as the coefficients of the trigonometric interpolation polynomial to $f$ at the nodes $t_{\mu}$, $\mu=0, \pm 1, \pm 2, \ldots$, one expects that the Fourier coefficients $c_{j}(f), j \in \mathbf{Z}$, are well approximated by the discrete Fourier coefficients. This can be shown for the values $d_{j}^{(n)}(f),|j| \leqslant n / 2$, if the function $f$ satisfies some regularity conditions. It is well known that

$$
c_{j}(f) \rightarrow 0 \text { as }|j| \rightarrow \infty
$$

Received March 13, 1980; revised December 15, 1980 and March 10, 1981.

1980 Mathematics Subject Classification. Primary 42A10, 65D10. 
if $f$ is integrable; there are more precise asymptotic results if one assumes stronger regularity conditions on $f$. But, because of the $n$-periodicity

$$
d_{j+\mu n}^{(n)}(f)=d_{j}^{(n)}(f), \quad \mu \in \mathbf{Z},
$$

of the sequence of discrete Fourier coefficients, this asymptotic behavior of the sequence $\left\{c_{j}(f)\right\}_{j=0, \pm 1, \pm 2, \ldots}$ is not simulated, and, indeed, $\left\{d_{j}^{(n)}(f)\right\}_{j=0, \pm 1, \pm 2, \ldots}$ cannot be the sequence of Fourier coefficients of any integrable function except in the trivial case $d_{j}^{(n)}(f)=0, j=0, \pm 1, \pm 2, \ldots$ This suggests a transformation of the sequence $\left\{d_{j}^{(n)}(f)\right\}_{j=0, \pm 1, \pm 2, \ldots}$, with the aid of a suitable limitation process, so that the asymptotic behavior of the resulting sequence $\left\{\hat{c}_{j}(f)\right\}_{j=0, \pm 1, \pm 2, \ldots}$, corresponds better to that of the sequence $\left\{c_{j}(f)\right\}_{j=0, \pm 1, \pm 2, \ldots}$. The function $\hat{f}$, whose Fourier coefficients are the $\hat{c}_{j}(f)$, should be an optimal approximation to $f$ in the sense of some norm.

We denote by $c, \hat{c}, \tilde{c}$ the two-sided infinite sequences of complex numbers

$$
\begin{aligned}
& c=\left\{\ldots, c_{-2}(f), c_{-1}(f), c_{0}(f), c_{1}(f), c_{2}(f), \ldots\right\}, \\
& \hat{c}=\left\{\ldots, \hat{c}_{-2}(f), \hat{c}_{-1}(f), \hat{c}_{0}(f), \hat{c}_{1}(f), \hat{c}_{2}(f), \ldots\right\}, \\
& \tilde{c}=\left\{\ldots, d_{-2}^{(n)}(f), d_{-1}^{(n)}(f), d_{0}^{(n)}(f), d_{1}^{(n)}(f), d_{2}^{(n)}(f), \ldots\right\},
\end{aligned}
$$

and by

$$
A=\left(a_{\nu \mu}\right)_{\nu, \mu=-\infty}^{\infty}
$$

an infinite matrix by which the sequence $\hat{c}$ is generated according to

$$
\hat{c}_{\nu}(f)=\sum_{k=-\infty}^{\infty} a_{\nu k} d_{k}^{(n)}(f), \quad \nu=0, \pm 1, \pm 2, \ldots
$$

We assume that $\hat{c} \in l_{2}$. The functions

$$
\begin{aligned}
& f \sim \sum_{j=-\infty}^{\infty} c_{j}(f) \exp (i j \cdot), \\
& \hat{f} \sim \sum_{j=-\infty}^{\infty} \hat{c}_{j}(f) \exp (i j \cdot), \\
& p=\sum_{j=0}^{n-1} d_{j}^{(n)}(f) \exp (i j \cdot),
\end{aligned}
$$

are associated with the sequences considered above. The sequence transformation which we consider is especially simple if $A$ has diagonal or band form. According to a result of Gautschi and Reinsch (cf. Gautschi [4]) this is the case if

$$
\hat{c}_{j}(f)=c_{j}\left(P_{n} f\right), \quad j=0, \pm 1, \pm 2, \ldots,
$$

with a translation invariant, respectively $r$-translation invariant, linear operator $\boldsymbol{P}_{n}$ which depends only on the data $f_{\mu}, \mu=0, \pm 1, \pm 2, \ldots$ For a translation invariant operator $P_{n}$ of the considered type, there exist attenuation factors $\tau_{j}^{(n)}, j=$ $0, \pm 1, \pm 2, \ldots$, so that

$$
c_{j}\left(P_{n} f\right)=\tau_{j}^{(n)} d_{j}^{(n)}(f), \quad j=0, \pm 1, \pm 2, \ldots,
$$

where $\tau_{j}^{(n)}$ depends only on $P_{n}$ but not on $f$. 
For an absolutely continuous and $2 \pi$-periodic function $\sigma$ with nonvanishing discrete Fourier coefficients $d_{k}^{(n)}(\sigma), k=0, \ldots, n-1$, it follows that

$$
\tau_{j}^{(n)}=\frac{c_{j}\left(P_{n} \sigma\right)}{d_{j}^{(n)}(\sigma)}, \quad j=0, \pm 1, \pm 2, \ldots
$$

A function $\sigma$ with

$$
\sigma=P_{n} \sigma
$$

may be considered as the generating function of the attenuation factors according to

$$
\tau_{j}^{(n)} \equiv \tau_{j}^{(n)}(\sigma)=\frac{c_{j}(\sigma)}{d_{j}^{(n)}(\sigma)}, \quad j=0, \pm 1, \pm 2, \ldots
$$

It is obvious that the asymptotic behavior of the sequence $\left\{\tau_{j}^{(n)}\right\}_{j=-\infty}^{\infty}$ depends on the smoothness of $\sigma$ and that the sequence $\left\{\hat{c}_{j}(f)\right\}_{j=-\infty}^{\infty}$ behaves asymptoticaliy for $|j| \rightarrow \infty$ according to the smoothness properties of $\sigma$. In order to obtain a good approximation to $f$ it was proposed by several authors to generate $P_{n} f$ by interpolation with polynomial splines on uniform meshes. A survey of the known results may be found in the work of Gautschi [4]. In this paper we also consider other interpolation functions, especially rational functions of $\exp (i \cdot)$. First we have to consider the corresponding interpolation problem which has the essential property that the associated coefficient matrix is of discrete convolution type.

As linear systems of that type may be solved with the aid of discrete Fourier transformation, we can formulate conditions which guarantee the unique solvability of the interpolation problem. Moreover, we can derive several representations of the interpolating function (Lagrange and discrete Fourier type). The interpolation problem generated by special kernels (especially reproducing kernels of certain Hilbert spaces) is considered in detail. Here the kernel and the functions generated from the kernel by shift are chosen as the basis of the interpolation space. In this context interesting examples are the polynomial splines and certain spaces of periodic analytic functions. The attenuation factors may be constructed with the aid of the Fourier expansion of the generating function $\sigma$. In this way we get the known attenuation factors for polynomial splines. On the other hand, it seems to be new that one may construct in the same manner attenuation factors for analytic splines.

2. Interpolation on Uniform Meshes. Let $\sigma: \mathbf{R} \rightarrow \mathbf{R}$ be a $2 \pi$-periodic, absolutely continuous initial function, which we consider in the following as the "generating function" of an interpolation problem $P_{n}$. If the almost everywhere existing derivative $\sigma^{\prime}$ is also quadratically integrable, the convergence of the Fourier series of $\sigma$ is absolute and uniform. We choose the points

$$
t_{j+n k}=\frac{2 \pi j}{n}+2 k \pi, \quad j=0, \ldots, n-1, k=0, \pm 1, \pm 2, \ldots,
$$

as the nodes of an interpolation problem and define the linear space of interpolating functions by

$$
V=\operatorname{span}\left\{\sigma_{j} \mid j=0, \ldots, n-1\right\}
$$


where $\sigma_{j}$ results from $\sigma$ by a $t_{j}$-shift according to

$$
\sigma_{j}=\sigma\left(\cdot-t_{j}\right), \quad j=0, \ldots, n-1 .
$$

We point out that for the interpolation process considered one has trivially $\sigma=P_{n} \sigma$.

An interpolating function

$$
g=\sum_{j=0}^{n-1} \alpha_{j} \sigma\left(\cdot-t_{j}\right)
$$

of the given data $f_{l+n k}, l=0, \ldots, n-1, k=0, \pm 1, \pm 2, \ldots$, is then defined by the linear system

$$
\sum_{j=0}^{n-1} \alpha_{j} \sigma\left(t_{l}-t_{j}\right)=f_{l}, \quad l=0, \ldots, n-1 .
$$

Criteria for the unique solvability of this system may be obtained with the aid of discrete Fourier analysis. (Here and in the sequel we use the notation and results from the paper of Henrici on fast Fourier algorithms [7].) If we denote by $\alpha, s$, and $f$ the sequences

$$
\begin{aligned}
\alpha & =\left(\ldots, \alpha_{n-1}, \alpha_{0}, \alpha_{1}, \ldots, \alpha_{n-1}, \alpha_{0}, \ldots\right), \\
s & =\left(\sigma\left(t_{j}\right)\right)_{j=-\infty}^{\infty}, \\
f & =\left(f_{l}\right)_{l=-\infty}^{\infty},
\end{aligned}
$$

the linear system (2.1) is of discrete convolution type

$$
\alpha * s=f .
$$

Taking the discrete Fourier transform on both sides of $(2.2)$ we get $[7$, p. 508]

$$
n \mathscr{F}_{n} \alpha \cdot \mathscr{F}_{n} s=\mathscr{F}_{n} f
$$

here the product on the left is understood in the sense of a Hadamard-product. Obviously the sequence $\mathscr{F}_{n} \alpha$ and then the sequence $\alpha$ itself is uniquely determined if and only if

$$
\left(\mathscr{F}_{n} s\right)_{k} \neq 0, \quad k=0, \ldots, n-1 .
$$

As $\left(\mathscr{F}_{n} s\right)_{k}$ is nothing but the discrete Fourier coefficient $d_{k}^{(n)}(\sigma)$, we get

THEOREM 2.1. The interpolation problem generated by $\sigma$ has a unique solution if and only if none of the discrete Fourier coefficients $d_{k}^{(n)}(\sigma), k=0, \ldots, n-1$, vanishes.

As the discrete Fourier coefficients $d_{k}^{(n)}(\sigma), k=0, \ldots, n-1$, may be expressed by the Fourier coefficients of $\sigma$, we get

$$
d_{k}^{(n)}(\sigma)=\sum_{\nu=-\infty}^{\infty} c_{k+\nu n}
$$

if $\sigma$ has the Fourier expansion

$$
\sigma(x)=\sum_{l=-\infty}^{\infty} c_{l} \exp (i l x), \quad c_{l}=\bar{c}_{-l}, \quad l \in \mathbf{Z} .
$$

So we get from Theorem 2.1 the conditions

$$
d_{k}^{(n)}(\sigma)=c_{k}+\sum_{\rho=1}^{\infty}\left\{\bar{c}_{\rho n-k}+c_{\rho+n k}\right\} \neq 0, \quad k=0, \ldots, n-1,
$$


which guarantee a unique solution of the interpolation problem. In our subsequent considerations the case of only real and nonnegative Fourier coefficients will play a dominant role. Here we get

COROLlaRY 2.2. If the Fourier coefficients $c_{l}, l=0, \pm 1, \pm 2, \ldots$ of the generating function $\sigma$ are all real and nonnegative, the interpolation problem generated by $\sigma$ has a unique solution if and only if there exists at least one index $l_{k}$ with $c_{l_{k}} \neq 0$ in every congruence class $n \mathbf{Z}+k, k=0, \ldots, n-1$.

3. Construction of Interpolating Functions. If the interpolation problem generated by $\sigma$ has a unique solution, there exist Lagrange functions $l_{j}, j=0, \ldots, n-1$, determined by the conditions

$$
l_{j}\left(t_{k}\right)=\delta_{j k}, \quad 0 \leqslant j, k \leqslant n-1 .
$$

Obviously

$$
l_{j+1}=l_{j}\left(\cdot-\frac{2 \pi}{n}\right), \quad j=0, \ldots, n-2,
$$

and therefore the Lagrange functions are known if the coefficients $\alpha_{\nu}, \nu=0, \ldots$, $n-1$, of

$$
l_{0}=\sum_{\nu=0}^{n-1} \alpha_{\nu} \sigma\left(\cdot-t_{\nu}\right)
$$

are determined from the linear system

$$
\sum_{\nu=0}^{n-1} \alpha_{\nu} \sigma\left(t_{k}-t_{\nu}\right)= \begin{cases}1, & \text { if } k=0, \\ 0, & \text { if } 1<k<n-1 .\end{cases}
$$

This is nothing else but

$$
\alpha * s=e_{0} .
$$

Taking the discrete Fourier transform, we get

$$
n \mathscr{F}_{n} \alpha \cdot \mathscr{F}_{n} s=\mathscr{F}_{n} e_{0}=\left(\ldots, \frac{1}{n}, \frac{1}{n}, \ldots, \frac{1}{n}, \ldots\right),
$$

and as

$$
\left(\mathscr{F}_{n} s\right)_{k} \neq 0, \quad k=0, \ldots, n-1,
$$

there follows

$$
\left(\mathscr{F}_{n} \alpha\right)_{k}=\frac{1}{n^{2}} \frac{1}{\left(\mathscr{F}_{n} s\right)_{k}}, \quad k=0, \ldots, n-1 .
$$

Inverting the Fourier transform, we get

$$
\alpha_{\nu}=\frac{1}{n} \sum_{\rho=0}^{n-1} \frac{\omega^{\rho \nu}}{\sum_{\lambda=0}^{n-1} \sigma\left(\frac{2 \pi}{n} \lambda\right) \omega^{-\rho \lambda}}, \quad \nu=0, \ldots, n-1 .
$$

Thus the interpolating function $p_{n}$ has the Lagrange form

$$
p_{n}=\sum_{j=0}^{n-1} f_{j} l_{0}\left(\cdot-\frac{2 \pi}{n} j\right)
$$

with $l_{0}$ from (3.3) and (3.9). 
As the Lagrange form is not optimal in several respects, one is interested in more favorable representations. For the spline interpolation problem on uniform meshes, Golomb [5] has considered that basis of the interpolation space which is obtained by interpolation of the exponential functions $\exp (i \nu \cdot), \nu=0, \ldots, n-1$. We apply this concept in our general context.

We start with the functions

$$
b_{\nu}=\sum_{\rho=0}^{n-1} \alpha_{\nu \rho} l_{0}\left(\cdot-\frac{2 \pi}{n} \rho\right), \quad \nu=0, \ldots, n-1,
$$

defined by the interpolation conditions

$$
b_{\nu}\left(\frac{2 \pi}{n} \mu\right)=\exp \left(\frac{2 \pi i}{n} \nu \mu\right), \quad \mu=0, \pm 1, \pm 2, \ldots
$$

Obviously

$$
\alpha_{\nu \rho}=\omega^{\nu \rho}
$$

and $b_{0}, \ldots, b_{n-1}$ is a basis of the linear space of interpolating functions. The basis functions $b_{p}, \nu=0, \ldots, n-1$, satisfy the discrete orthogonality relation

$$
\begin{aligned}
\frac{1}{n} \sum_{\rho=0}^{n-1} b_{\nu}\left(\frac{2 \pi}{n} \rho\right) \overline{b_{\mu}\left(\frac{2 \pi}{n} \rho\right)} & \\
=\frac{1}{n} \sum_{\rho=0}^{n-1} \exp \left(\frac{2 \pi i}{n}(\nu-\mu) \rho\right) & = \begin{cases}1, & \text { if } \nu=\mu, \\
0, & \text { else. }\end{cases}
\end{aligned}
$$

This implies the relation

$$
d_{\mu}^{(n)}\left(b_{\nu}\right)= \begin{cases}1, & \text { if } \nu=\mu \\ 0, & \text { else. }\end{cases}
$$

We see that the functions $b_{0}, \ldots, b_{n-1}$ and the linear functionals $d_{0}^{(n)}, \ldots, d_{n-1}^{(n)}$ are mutually orthogonal bases of the space $V$ of interpolating functions and its dual space. For

$$
p=\sum_{j=0}^{n-1} d_{j}^{(n)}(f) b_{j}
$$

it follows that

$$
d_{k}^{(n)}(p)=d_{k}^{(n)}(f), \quad k=0, \ldots, n-1 ;
$$

$p$ "interpolates" the discrete Fourier coefficients. On the other hand, because of the numerical Fourier synthesis, one has

$$
\begin{aligned}
p\left(t_{k}\right) & =\sum_{j=0}^{n-1} d_{j}^{(n)}(f) b_{j}\left(t_{k}\right) \\
& =\sum_{j=0}^{n-1} d_{j}^{(n)}(f) \omega^{j k}=f_{k}, \quad k=0, \ldots, n-1 .
\end{aligned}
$$

COROLlaRY 3.1. The interpolating function has a representation of discrete Fourier type

$$
p=\sum_{j=0}^{n-1} d_{j}^{(n)}(f) b_{j}
$$




$$
b_{j}=\sum_{\rho=0}^{n-1} \omega^{j \rho} l_{0}\left(\cdot-\frac{2 \pi}{n} \rho\right),
$$

where $l_{0}$ is the Lagrange function from (3.3).

If the expression (3.3), (3.9) for $l_{0}$ is used in (3.20), one obtains a representation of the $b_{j}$ in terms of the function $\sigma$ :

COROLlaRY 3.2. The interpolating function has the representation of discrete Fourier type

$$
\begin{aligned}
p & =\sum_{\nu=0}^{n-1} d_{\nu}^{(n)}(f) b_{\nu} \\
b_{\nu} & =\frac{1}{n d_{\nu}^{(n)}(\sigma)} \sum_{\mu=0}^{n-1} \omega^{\nu \mu_{\sigma}}\left(\cdot-\frac{2 \pi}{n} \mu\right), \quad \nu=0, \ldots, n-1 .
\end{aligned}
$$

In case of

$$
\sigma=\frac{1}{n} \sum_{\rho=0}^{n-1} \exp (i \rho \cdot)
$$

this is obviously the formula

$$
p=\sum_{\nu=0}^{n-1} d_{\nu}^{(n)}(f) \exp (i \nu \cdot)
$$

of trigonometric interpolation.

4. Examples. In this section we consider some examples, especially interpolation by polynomial and analytic splines.

Example 4.1. We start with the generating function

$$
\sigma=1+\sum_{\rho=-\infty}^{\infty} \frac{\exp (i \rho \cdot)}{|\rho|^{2 s}}=1+2 \sum_{\rho=1}^{\infty} \frac{\cos (\rho \cdot)}{\rho^{2 s}},
$$

where $s \in \mathbf{R}$ with $s>3 / 4$. (' indicates that the index 0 is omitted.) For a later application in connection with attenuation factors we calculate the discrete Fourier coefficients of $\sigma$ as

$$
\begin{aligned}
d_{\nu}^{(n)}(\sigma)= & \sum_{\tau=-\infty}^{\infty} c_{\nu+\tau n}=\sum_{\tau=-\infty}^{\infty}|\nu+\tau n|^{-2 s} \\
= & \sum_{\tau=1}^{\infty}\left\{(\tau n-\nu)^{-2 s}+(\tau n+\nu)^{-2 s}\right\}, \quad \nu=1, \ldots, n-1, \\
& d_{0}^{(n)}(\sigma)=1+2 \sum_{\tau=1}^{\infty}(\tau n)^{-2 s}=1+2 n^{-2 s \xi}(2 s) .
\end{aligned}
$$

If $1<s \in \mathbf{N}$, we may consider the Hilbert space $E$ of $(s-1)$-times continuously differentiable functions $f$ with an absolutely continuous $(s-1)$ th derivative for which $f^{(s)} \in L_{2 \pi}^{2}$. $E$ may be seen as the subspace of $L_{2 \pi}^{2}$, whose elements have Fourier coefficients which decrease asymptotically at least like $|j|^{-s}$ for $|j| \rightarrow \infty$; more precisely

$$
E=\left\{f \in L_{2 \pi}^{2}: \sum_{j=-\infty}^{\infty}|j|^{2 s}\left|c_{j}(f)\right|^{2}<\infty\right\}
$$


with norm

$$
\|f\|:=\left\{\left|c_{0}(f)\right|^{2}+\sum_{j=-\infty}^{\infty}|j|^{2 s}\left|c_{j}(f)\right|^{2}\right\}^{1 / 2}
$$

By $\left\{e_{\rho}\right\}_{\rho=-\infty}^{\infty}$, with

$$
e_{\rho}= \begin{cases}1, & \rho=0 \\ \frac{\exp (i \rho \cdot)}{|\rho|^{s}}, & \rho= \pm 1, \pm 2, \ldots,\end{cases}
$$

we get a complete orthonormal system of $E$, and $\sigma$ from (4.1) is the associated reproducing kernel. If we use $\sigma$ as the generating function of an interpolation problem, the interpolants are splines.

Remark. The interpolating splines of Example 4.1 are closely related to, but not identical with those studied in detail by Golomb [5]. The difference results from the fact that $P_{n} 1=b_{0}$ (by (3.12) with $\nu=0$ ), while $S_{n} 1=1$ for Golomb's interpolation operator $S_{n}$. Clearly, $b_{0} \neq 1$, but $b_{1}, \ldots, b_{n-1}$ are the same as in [5]; this can be seen from comparing (3.22), (4.1) with [5, Eq. (5.8)]. If $d_{0}^{(n)}(f)=0$, then $P_{n} f$ is a linear combination of $b_{1}, \ldots, b_{n-1}$ alone (see (3.21)), and so is $S_{n} f$ (see [5, Eq. (5.6)]). Hence in this case $P_{n} f=S_{n} f$.

Example 4.2. Starting with

$$
\sigma=\sum_{\rho=-\infty}^{\infty} \frac{\exp (i \rho \cdot)}{R^{2|\rho|}}=\frac{R^{4}-1}{R^{4}-2 R^{2} \cos (\cdot)+1}, \quad R>1,
$$

we get another interesting example. It is seen that $\sigma$ is a rational function of $\exp (i \cdot)$ and has simple poles at $z_{k}, \bar{z}_{k}, k \in \mathbf{Z}$, where $z_{k}=2 i \log R+2 k \pi$. We point out that $\sigma$ is the reproducing kernel of the subspace $E \subset L_{2 \pi}^{2}$ with geometrically decreasing Fourier coefficients; more precisely

$$
E=\left\{f \in L_{2 \pi}^{2}: \sum_{j=-\infty}^{\infty} R^{2|j|}\left|c_{j}(f)\right|^{2}<\infty, R>1\right\}
$$

with norm

$$
\|f\|=\left\{\sum_{j=-\infty}^{\infty} R^{2|j|}\left|c_{j}(f)\right|^{2}\right\}^{1 / 2} .
$$

It is well known that the functions of $E$ have analytic extensions to the strip

$$
S_{a}:=\{z|z=x+i y,| y \mid<a:=\log R\} .
$$

The interpolants generated by $\sigma$ are the analytic splines introduced by Golomb in [6, Section 8]. Here we get the discrete Fourier coefficients

$$
d_{\nu}^{(n)}(\sigma)=\sum_{\tau=-\infty}^{\infty} R^{-2|\nu+\tau n|}=\frac{R^{-2|\nu|}\left(1+R^{-2 n+4|\nu|}\right)}{1-R^{-2 n}}, \quad|\nu|<\frac{n}{2} .
$$

Example 4.3. The examples considered above can be generalized in the following way: Let $E$ be a Hilbert space of complex-valued functions which are real-valued on the real line, $2 \pi$-periodic and analytic on a domain $D \supseteq \mathbf{R}$ of the complex plane. We assume $\exp (i \nu \cdot) \in E, \nu \in \mathbf{Z}$, and that $E$ has a reproducing kernel $\sigma$ of convolution type. It is easily seen that the Fourier coefficients of $\sigma$ are all real and 
strictly positive, so that $\sigma$ may be taken as the generating function of an interpolation problem. It is well known that one obtains in this way a "smoothest" interpolation of the given data in the sense that an interpolant of the form $p=\sum_{j=0}^{n-1} \alpha_{j} \sigma\left(\cdot-t_{j}\right)$ has minimum norm with respect to all elements of $E$ which interpolate the data (cf. Meschkowski [10, p. 140]).

In this context we recall the method proposed by Kress [9] and Knauff-Kress [8] to define function spaces of the considered type with the aid of area or contour integrals. As an example let $\tilde{E}$ be the space of periodic functions, holomorphic in a strip $S_{a}$, for which

$$
\lim _{\alpha \rightarrow a-0} \iint_{\substack{0<x<2 \pi \\|y|<\alpha}}|f(z)|^{2} d x d y<\infty
$$

(cf. Kress [9]). $\tilde{E}$ possesses the reproducing kernel

$$
\tilde{\sigma}=\sum_{\rho=-\infty}^{\infty}\left(\frac{\rho}{2 \pi \sinh 2 \rho a}\right)^{1 / 2} \exp (i \rho \cdot)
$$

which may be represented with the aid of elliptic functions (cf. Bergman [1, p. 10]). For moderately large values of $a$, the Fourier coefficients of $\tilde{\sigma}$ and of $\sigma$ from Example 4.2 do not differ very much. Therefore, in general, the simple kernel $\sigma$ is preferable.

5. Evaluation of Attenuation Factors. We pointed out that a generating function $\sigma$ of an interpolation problem with

$$
d_{j}^{(n)}(\sigma) \neq 0, \quad j=0, \ldots, n-1,
$$

offers the possibility to define attenuation factors

$$
\tau_{j}^{(n)}(\sigma)=\frac{c_{j}(\sigma)}{d_{j}^{(n)}(\sigma)}, \quad j=0, \pm 1, \pm 2, \ldots,
$$

such that

$$
c_{j}\left(P_{n} f\right)=\tau_{j}^{(n)}(\sigma) d_{j}^{(n)}(f), \quad j=0, \pm 1, \pm 2, \ldots
$$

Since

$$
d_{j}^{(n)}(\sigma)=\sum_{\rho=-\infty}^{\infty} c_{j+\rho n}(\sigma)
$$

we get

$$
\tau_{j}^{(n)}(\sigma)=\frac{c_{j}(\sigma)}{\sum_{\rho=-\infty}^{\infty} c_{j+\rho n}(\sigma)}, \quad j=0, \pm 1, \pm 2, \ldots
$$

Setting $x:=j / n$ and $\tau^{(n)}(\sigma ; x):=\tau_{j}^{(n)}(\sigma)$, this yields

$$
\tau^{(n)}(\sigma ; x)=\frac{c_{x n}(\sigma)}{\sum_{\rho=-\infty}^{\infty} c_{n(x+\rho)}(\sigma)}
$$


where for some $\sigma$ the function $\tau^{(n)}(\sigma ; \cdot)$ is independent of $n$. The extreme case of trigonometric interpolation is characterized by

$$
\tilde{\boldsymbol{\sigma}}=\sum_{j=-\infty}^{\infty} c_{j} \exp (i j \cdot)
$$

where

$$
\begin{aligned}
& c_{j}=\frac{1}{n}, \quad j=-\frac{n-1}{2}, \ldots, \frac{n-1}{2}, \quad \text { if } n \text { odd, } \\
& c_{j}= \begin{cases}\frac{1}{n}, & j=-\frac{n}{2}+1, \ldots, \frac{n}{2}-1, \quad \text { if } n \text { even, } \\
\frac{1}{2 n}, & j=-\frac{n}{2} \text { and } j=\frac{n}{2},\end{cases}
\end{aligned}
$$

and $c_{j}=0$, for the remaining $j$. Here we have the special attenuation factors

$$
\tau^{(n)}(\tilde{\sigma} ; x)= \begin{cases}0, & \text { if }|x|>\frac{1}{2}, \\ 1, & \text { if }|x|<\frac{1}{2}, \\ 0, & \text { if }|x|=\frac{1}{2} \text { and } n \text { odd } \\ \frac{1}{2}, & \text { if }|x|=\frac{1}{2} \text { and } n \text { even. }\end{cases}
$$

For other $\sigma$ one may consider the corresponding function $\tau^{(n)}(\sigma ; \cdot)$ as an approximation of $\tau^{(n)}(\tilde{\sigma} ; \cdot)$. In every case, $\tau^{(n)}(\sigma ; \cdot)$ has compact support if $\sigma$ is a trigonometric polynomial of any degree. We now let $\sigma$ be one of the reproducing kernels of Section 4. In case of the kernel

$$
\sigma=1+\sum_{\rho=-\infty}^{\infty} \frac{\exp (i \rho \cdot)}{|\rho|^{2 s}}
$$

of Example 4.1, one gets the attenuation factors

$$
\tau_{j}^{(n)}(\sigma)= \begin{cases}\frac{1}{d_{0}^{(n)}(\sigma)}, & j=0, \\ \frac{1}{|j|^{2 s} d_{j}^{(n)}(\sigma)}, & j \neq 0 .\end{cases}
$$

Substituting the expressions for $d_{j}^{(n)}(\sigma)$ from (4.2) and (4.3), there follows

$$
\tau_{j+\mu n}^{(n)}(\sigma)= \begin{cases}\frac{1}{1+2 n^{-2 s}(2 s)}, & j=\mu=0, \\ \frac{1}{|\mu|^{2 s} n^{2 s}\left(1+2 n^{-2 s}(2 s)\right)}, & j=0, \mu \in \mathbf{Z} \backslash\{0\}, \\ \left\{\sum_{\rho=-\infty}^{\infty}\left(\frac{j+\mu n}{j+\rho n}\right)^{2 s}\right\}^{-1}, & 1 \leqslant j \leqslant n-1, \mu \in \mathbf{Z} .\end{cases}
$$

As expected, these are for $s \in \mathbf{N}$ the attenuation factors of polynomial splines which first were calculated by Eagle in 1928 (cf. Collatz and Quade [2], Ehlich [3], Gautschi [4]). 
TABLE 1

Numerical values of attenuation factors generated

$$
\text { by } \sigma(x)=\sum_{\rho--\infty}^{\infty} R^{-2|\rho|} \exp (i \rho x)
$$

\begin{tabular}{|c|c|c|c|c|c|}
\hline \multirow[b]{2}{*}{$x$} & \multicolumn{5}{|c|}{$R^{-2 n}$} \\
\hline & $10^{-6}$ & $10^{-8}$ & $10^{-10}$ & $10^{-12}$ & $10^{-14}$ \\
\hline 0 & 0.999999 & 0.999999 & 0.999999 & 0.999999 & 0.999999 \\
\hline 0.1 & 0.999984 & 0.999999 & 0.999999 & 0.999999 & 0.999999 \\
\hline 0.2 & 0.999749 & 0.999984 & 0.999999 & 0.999999 & 0.999999 \\
\hline 0.3 & 0.996035 & 0.999369 & 0.999900 & 0.999984 & 0.999997 \\
\hline 0.4 & 0.940649 & 0.975450 & 0.990099 & 0.996035 & 0.998418 \\
\hline 0.41 & 0.923211 & 0.964964 & 0.984398 & 0.993129 & 0.996989 \\
\hline 0.42 & 0.901187 & 0.950136 & 0.975497 & 0.988120 & 0.994279 \\
\hline 0.43 & 0.873710 & 0.929491 & 0.961713 & 0.979535 & 0.989154 \\
\hline 0.44 & 0.839951 & 0.901187 & 0.940649 & 0.964964 & 0.979535 \\
\hline 0.45 & 0.799240 & 0.863193 & 0.909091 & 0.940649 & 0.961713 \\
\hline 0.46 & 0.751241 & 0.813612 & 0.863193 & 0.901187 & 0.929490 \\
\hline 0.47 & 0.696129 & 0.751241 & 0.799240 & 0.839951 & 0.873710 \\
\hline 0.48 & 0.634743 & 0.676302 & 0.715253 & 0.751241 & 0.784054 \\
\hline 0.49 & 0.568641 & 0.591076 & 0.613137 & 0.634743 & 0.655821 \\
\hline 0.5 & 0.500000 & 0.500000 & 0.500000 & 0.500000 & 0.500000 \\
\hline 0.51 & 0.431359 & 0.408924 & 0.386863 & 0.365257 & 0.344179 \\
\hline 0.52 & 0.365257 & 0.323698 & 0.284747 & 0.248759 & 0.215946 \\
\hline 0.53 & 0.303871 & 0.248759 & 0.200760 & 0.160049 & 0.126290 \\
\hline 0.54 & 0.248759 & 0.186388 & 0.136807 & 0.098813 & 0.070509 \\
\hline 0.55 & 0.200760 & 0.136807 & 0.090909 & 0.059351 & 0.038287 \\
\hline 0.56 & 0.160049 & 0.098813 & 0.059351 & 0.035036 & 0.020465 \\
\hline 0.57 & 0.126290 & 0.070509 & 0.038287 & 0.020466 & 0.010846 \\
\hline 0.58 & 0.098813 & 0.049864 & 0.024503 & 0.011880 & 0.005721 \\
\hline 0.59 & 0.076789 & 0.035036 & 0.015602 & 0.006871 & 0.003011 \\
\hline 0.6 & 0.059351 & 0.024503 & 0.009901 & 0.003965 & 0.001582 \\
\hline 0.7 & 0.003965 & 0.000631 & 0.000100 & 0.000016 & 0.000003 \\
\hline 0.8 & 0.000251 & 0.000016 & 0.000001 & 0.000000 & 0.000000 \\
\hline 0.9 & 0.000016 & 0.000000 & 0.000000 & 0.000000 & 0.000000 \\
\hline 1.0 & 0.000001 & 0.000000 & 0.000000 & 0.000000 & 0.000000 \\
\hline
\end{tabular}

We point to the fact that

$$
\tau_{\mu n}^{(n)}(\sigma) \neq \begin{cases}1, & \mu=0 \\ 0, & \mu \in \mathbf{Z} \backslash\{0\}\end{cases}
$$

in contrast to the values given in [3] and [4]. This difference disappears in the limit as $n \rightarrow \infty$. More precisely, let $\tau_{\infty}(\sigma ; x)$ denote the limit of $\tau_{j}^{(n)}(\sigma)$, as both $n$ and $j=j(n)$ go to infinity, such that

$$
\lim _{n \rightarrow \infty} j(n) / n=x, \quad 0 \leqslant x<1 .
$$


Then

$$
\tau_{\infty}(\sigma ; x+\mu)= \begin{cases}1, & x=\mu=0, \\ 0, & x=0, \mu \in \mathbf{Z} \backslash\{0\}, \\ \left\{\sum_{\rho=-\infty}^{\infty}\left(\frac{x+\mu}{x+\rho}\right)^{2 s}\right\}^{-1}, & 0<x<1, \mu \in \mathbf{Z} .\end{cases}
$$

In case of

$$
\sigma=\sum_{\rho=-\infty}^{\infty} \frac{\exp (i \rho \cdot)}{R^{2|\rho|}}
$$

of Example 4.2, we get the attenuation factors

$$
\begin{aligned}
\tau_{j+\mu n}^{(n)}(\sigma) & =\frac{1}{R^{2|j+\mu n|} \sum_{\rho=-\infty}^{\infty} R^{-2|j+\mu n|}} \\
& =\frac{1-R^{-2 n}}{R^{2|j+\mu n|-2|j|}\left(1+R^{-2 n+4|j|}\right)}, \quad j<\frac{n}{2}, \mu \in \mathbf{Z} .
\end{aligned}
$$

We set $x=j / n, k=R^{-2 n}$, and obtain

$$
\tau^{(n)}(x+\mu)=\frac{k^{|x+\mu|-|x|}(1-k)}{1+k^{1-2|x|}}, \quad|x|<\frac{1}{2}, \mu \in \mathbf{Z} .
$$

If $k \rightarrow 0$, one gets the attenuation factors $\tilde{\tau}(x)$ which belong to trigonometric interpolation. A comparison with the attenuation factors of polynomial splines shows that in the case of the above generating function $\tilde{\tau}$ is more sharply simulated (see Table 1 and Figure 1).

The same can be said about the kernel $\tilde{\sigma}$ from Example 4.3 which produces the attenuation factors

$$
\begin{aligned}
\tau^{(n)}(x+\mu)= & \left(\frac{x+\mu}{2 \pi \sinh 2(x+\mu) n a}\right)^{1 / 2} \\
& \cdot\left\{\sum_{\rho=-\infty}^{\infty}\left(\frac{x+\rho}{2 \pi \sinh 2(x+\rho) n a}\right)^{1 / 2}\right\}^{-1}, \quad|x|<\frac{1}{2}, \mu \in \mathbf{Z},
\end{aligned}
$$

and about the kernel

$$
\sigma=\sum_{\rho=-\infty}^{\infty} \frac{\exp (i \rho \cdot)}{\cosh (\rho a)}, \quad a>1,
$$

which was introduced by Achieser and Krein in connection with $n$-width problems of analytic functions (cf. [11, p. 196]). Here we have the attenuation factors

$$
\tau^{(n)}(x+\mu)=\frac{1}{\cosh ((x+\mu) n a)}\left\{\sum_{\rho=-\infty}^{\infty} \frac{1}{\cosh ((x+\rho) n a)}\right\}^{-1},
$$

$$
|x|<\frac{1}{2}, \mu \in \mathbf{Z} \text {. }
$$

It is seen that in (5.18) and (5.20) the term

$$
\kappa=\exp (-a n)
$$

plays a similar role as $k$ in (5.17). 


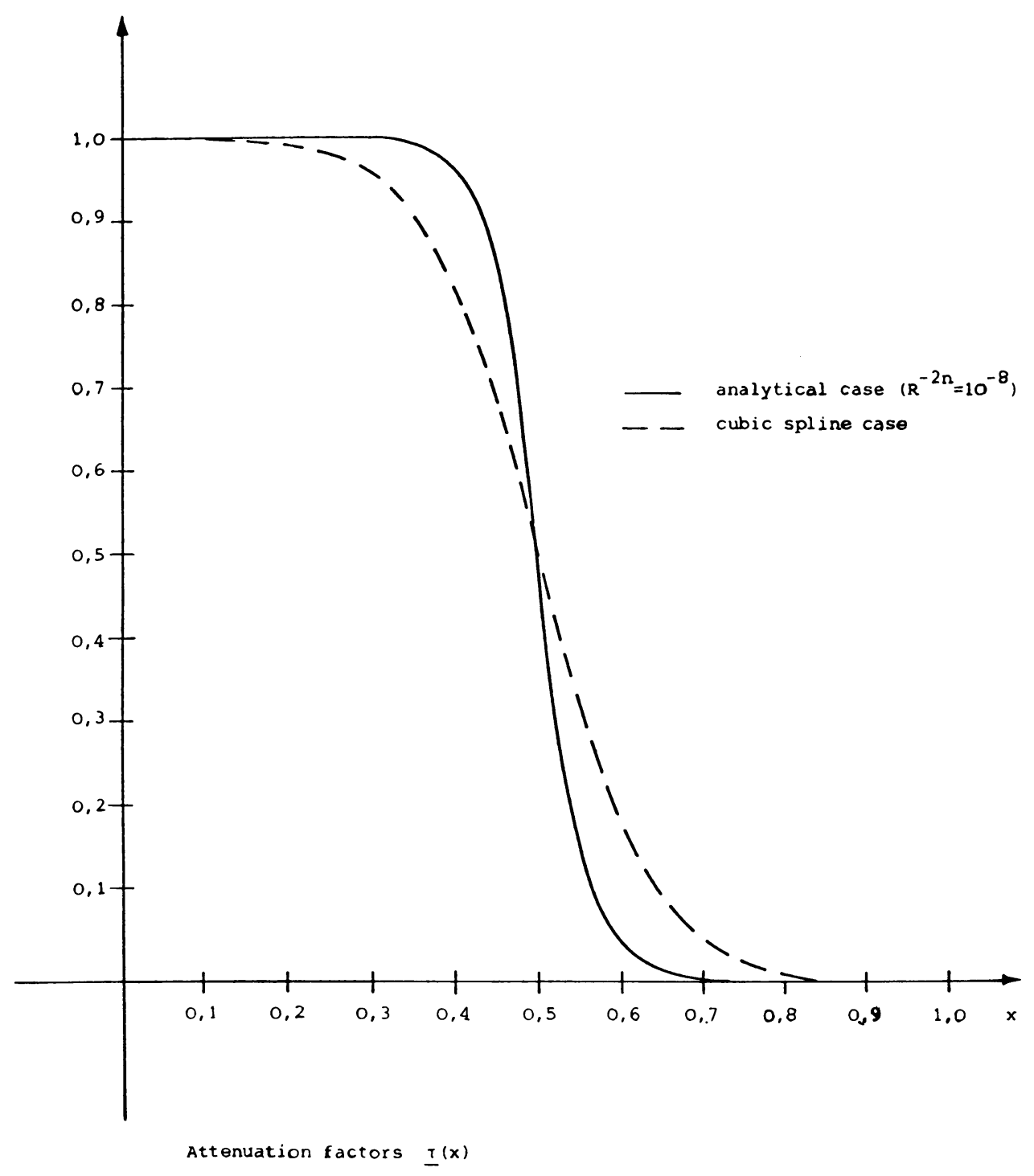

FIGURe 1

Acknowledgement. I am greatly indebted to the referee for his very helpful suggestions especially in connection with the proof of Theorem 2.1 .

Fachbereich Mathematik

Fernuniversität-Gesamthochschule

Postfach 940

D-5800 Hagen, West Germany

1. S. Bergman, The Kernel Function and Conformal Mapping, Math. Surveys, no. 5, Amer. Math. Soc., Providence, R. I., 1970.

2. L. Collatz \& W. QUADE, “Zur Interpolationstheorie der reellen periodischen Funktionen," $S$.-B. Preuss. Akad. Wiss. Phys.-Math. Kl., v. 30, 1938, pp. 383-429.

3. H. EHLICH, "Untersuchungen zur numerischen Fourieranalyse," Math. Z., v. 91, 1966, pp. $380-420$. 
4. W. GAUTSCH, "Attenuation factors in practical Fourier analysis," Numer. Math., v. 18, 1972, pp. 373-400.

5. M. GoLomB, “Approximation by periodic spline interpolants on uniform meshes," J. Approx. Theory, v. 1, 1968, pp. 26-65.

6. M. GoLOMB, "Interpolation operators as optimal recovery schemes for classes of analytic functions," Optimal Estimation in Approximation Theory (Ch. A. Micchelli and Th. J. Rivlin, Eds.), Plenum Press, New York and London, 1976.

7. P. Henrici, "Fast Fourier methods in computational complex analysis," SIAM Rev., v. 21, 1979, pp. 481-527.

8. W. KnaUfF \& R. KRess, "Optimale Approximation linearer Funktionale auf periodischen Funktionen," Numer. Math., v. 22, 1974, pp. 187-205.

9. R. KRESS, "Zur numerischen Integration periodischer Funktionen nach der Rochteckregel," Numer. Math., v. 20, 1972, pp. 87-92.

10. H. MeschKowsKI, Reihenentwicklungen in der mathematischen Physik, Bibliographisches Institut, Mannheim, 1963.

11. A. Schönhage, Approximationstheorie, de Gruyter, Berlin and New York, 1971. 